\title{
Tipping Point Pandemie?
}

\author{
Stefan Reinheimer · Susanne Robra-Bissantz
}

Angenommen: 9. Juni 2021

(C) Springer Fachmedien Wiesbaden GmbH, ein Teil von Springer Nature 2021

Ist die Covid-19-Pandemie ein Wendepunkt, wie es der neudeutsch formulierte Titel hinterfragt? Man beachte, dass wir am Ende ein Frage-, kein Ausrufezeichen gesetzt haben. Fordern die inzwischen gewonnenen Erkenntnisse über die Auswirkungen dieser Pandemie auf die Rolle der IT nicht eigentlich eindeutig das Exklamationszeichen? Zur Erklärung des Satzzeichens möchten wir Ihnen einen kleinen Einblick in die Historie und den Entscheidungsprozess für das Schwerpunktthema des Heftes 340 (oder offiziell: Heft Nr. 4 im 58. Jahrgang der HMD) geben, bevor Sie, wie gewohnt, einen kurzen Aus- und Einblick in das Heft und seine Beiträge erhalten:

Schwerpunktthemen der HMD werden im Herausgeberkreis definiert und terminiert. In unserer Herausgebersitzung Anfang Juli 2020 kamen wir zu der Überzeugung, dass eine globale Pandemie wie die, in der wir uns damals gerade befanden, Auswirkungen auf den Einsatz und die Bedeutung der IT haben würde. Interessanterweise sind wir damals noch davon ausgegangen, dass schon im Sommer desselben Jahres der Schrecken des Virus abgeklungen sein würde. Von Masken in OP- oder FFP2-Ausprägung, Quarantäne, Homeoffice, Ausgangssperre, Besuchsregelungen, Homeschooling und dem Zusammenbruch ganzer Wirtschaftszweige waren wir damals gedanklich noch sehr weit entfernt. Unsere Überzeugung war, dass sich Unternehmen und Hochschulen schon mit den damaligen Erfahrungen intensiv befassen und Konzepte entwickeln würden, um für zukünftige Pandemien gerüstet zu sein. Unsere Berechnung ermittelte das vierte Heft 2021 (August 2021) als geeigneten Zeitpunkt, um ca. ein Jahr Abstand von der Pandemie zu haben. Genug Zeit, um das

Stefan Reinheimer $(\bowtie)$

BIK GmbH, Äußere Sulzbacher Str. 16, 90489 Nürnberg, Deutschland

E-Mail: sr@bik.biz

Susanne Robra-Bissantz

Technische Universität Braunschweig, Universitätsplatz 2, 38106 Braunschweig, Deutschland

E-Mail: s.robra-bissantz@tu-bs.de 
Erfahrene zu verarbeiten und in Forschung und Praxis Erkenntnisse und Lösungsansätze zu erarbeiten und zu publizieren. Unglaublich, wie weit wir damals daneben lagen und wie unvorstellbar es für uns war, dass die Pandemie und ihre Wellen einen Impact hinterlassen würden, wie sie es dann tatsächlich tat. Also bereits unser damaliger Kenntnisstand legitimierte eine eigene Ausgabe der HMD. Hätten wir damals gewusst, was uns noch bevorstand, hätten wir mit Sicherheit ein Ausrufezeichen an das Ende des Titels gesetzt. Natürlich hätten wir dies bis zum Redaktionsschluss Mitte Juni 2021 noch ändern können, aber viel lieber haben wir das falsche Satzzeichen als warnende Erinnerung an unsere krasse Fehleinschätzung belassen und damit einen Aufhänger für dieses Editorial geschaffen. Ich denke, heute sind wir uns alle einig: die Pandemie WAR ein Tipping Point für die Informationstechnologie. Und hiermit kommen wir zu einem kurzen Überblick über die Beiträge des vorliegenden Heftes:

Fast alle Ausgaben der HMD beginnen inhaltlich mit einem sogenannten Überblicksbeitrag, der das Schwerpunktthema umreißt. Einen entsprechenden Beitrag konnten wir für das vorliegende Heft nicht gewinnen - vermutlich ist das Thema (noch) zu schwierig ein- und abgrenzbar. Im akademischen und Praxisumfeld war eine Draufsicht auf die IT in Zeiten einer globalen Pandemie wohl nicht opportun - man beschäftigt sich lieber mit einzelnen konkreten Anforderungen. Daher entschieden wir uns für einen alternativen Ansatz: wir baten die WKWI (Wissenschaftliche Kommission Wirtschaftsinformatik des Verbands der Hochschullehrer für Betriebswirtschaft) um eine Situations- und Erfahrungseinschätzung in Form eines Einwurfs - einem Beitragsformat, das keinem Review-Verfahren unterliegt, keine wissenschaftliche Bearbeitung eines Themas inklusive entsprechender Literaturarbeit erwartet, sondern subjektive Meinungen fordert und fördert. Für die WKWI übernahm der zum Zeitpunkt unserer Anfrage amtierende Vorsitzende, Prof. Rainer Alt (Universität Leipzig), diese Aufgabe und sicherte sich als Co-Autorin die Mit-Herausgeberin dieser HMD (Prof. Susanne Robra-Bissantz von der TU Braunschweig).

Die folgenden, insgesamt 15 Beiträge aus dem akademischen und Praxisumfeld starten mit dem Pflichtprogramm der Digitalisierung in der Pandemie: mit denjenigen Applikationen, die zum Management der Pandemie umgesetzt werden mussten. Dabei analysiert der Beitrag von Brendel et al. den europäischen Corona-AppMarkt anhand unterschiedlicher Architekturentscheidungen und Zimmermann et al. bieten einen fast schon intimen Einblick in die administrativen Kämpfe, die das Gesundheitsamt der Stadt Nürnberg ausfechten und die Entwicklung, die man mit zunehmender Pandemieausweitung nehmen musste.

Auch die nächsten zwei Beiträge zeigen auf, wie die Anforderung einer zunehmenden Digitalisierung einzelner Prozesse angenommen wird. So beschreibt Brechlin, dass auch die nun beschleunigte Transformation der Geschäftstätigkeit in Clouds eines wohl konzipierten Fahrplans und Changemanagements bedarf. Kortekamp et al. nehmen sich der zahnärztlichen Versorgung an und erarbeiten, welche Behandlungen wie und unter welchen Bedingungen diese im häuslichen Umfeld mit AR-Unterstützung auf Distanz umgesetzt werden können.

Der Artikel \#BrilliantBedient verallgemeinert die Sicht auf Dienstleistungen für Kunden und führt als wesentliche, digital zu unterstützende Aspekte die Wertschaf- 
fung, synchrone Interaktionen und Zusammenarbeit ein - die ebenso auch für Forschungsdienstleistungen relevant sind. Folgend wird der Gedanke der nachhaltig wertvollen Dienstleistung im Artikel von Butt et al. weitergeführt - sie analysieren, wie digitale Plattformen, hier zur Bottom-up-Partizipation, über Wandlungs- und Anpassungsfähigkeit auch in Krisen resilient sind.

Um auch in der Krise wertvolle Dienstleistungen oder Produkte anbieten zu können, bedarf es neuer Formen der Führung, Zusammenarbeit und Innovation im Unternehmen. Bergsleitner \& Rueckel betrachten zunächst, wie die Akzeptanz des Distance Working von Führungskräften gesteigert werden kann und erarbeiteten mit ihren Interviewpartnern, dass es zum Technikeinsatz insbesondere eines gesteigerten Vertrauens sowie einer geplanten, auch sozialen Kommunikation bedarf. Auch Ebert nimmt sich der verteilten Arbeit, hier in Teams, an und beschreibt in vielen Facetten deren Erfolgsfaktoren, die z. B. in einem guten Wissensmanagement, ergebnisorientierter Führung und Förderung von Soft Skills bestehen. Gleichwohl zeigt er auch auf, was die verteilte Arbeit mit dem Mitarbeiter macht - um darauf ebenfalls als Führungskraft reagieren zu können.

Die folgenden zwei Beiträge suchen, erarbeiten und nutzen Methoden für die $\mathrm{Zu}$ sammenarbeit von Mitarbeitern, in Workshop-Settings. Dabei entwickeln Waldhör \& Kubla eine Beurteilungsmethode, welche Kreativitätstechniken sich (am Beispiel eines Software-Entwicklers) für die kreative Arbeit auf Kommunikationsplattformen (wie z.B. Zoom) eignen - und setzen diese in einer Fallstudie ein. Lux \& Macgilchrist erarbeiten aus den Anforderungen postdigital, interaktiv und partizipativ mithilfe des Konzepts der Liberating Structures Workshop-Methoden, die selbst eine (kreative) Strategie-Entwicklung erlauben und so auch in eine post-Corona Zeit getragen werden können.

Mit ihrem Beitrag zum Blended Learning zeigen Kätzel \& Durst auf, wie sie einen gesamten, mitten in der Pandemie gestarteten Studiengang trotzdem erfolgreich durchführen und leiten daraus ähnliche Empfehlungen für Unternehmen wie in den vorausgehenden Artikeln ab, z. B. hinsichtlich der Relevanz von Soft Skills ihrer Mitarbeiter sowie eines Methodensets für Workshops.

Damit und mit drei weiteren Artikeln schlägt die vorliegende Ausgabe der HMD bereits die Brücke zu dem nächsten Herausgeberheft, das sich gezielt mit dem Lernen und Lehren in der Pandemie beschäftigt.

Grogorick und Lamprecht stellen in ihrem Artikel ACDC dar, wie Studierende mithilfe von Gamification und der Erstellung eigener, praxisnaher Lerninhalte zu einem höheren Lernerfolg gelangen. Das technisch sehr anspruchsvolles Multiagentensystem im schulischen Lernen von Gösling et al. ermöglicht individuelles Lernen in kooperativen Lernmethoden. Schließlich zeigen Voigt et al. auf, wie das gesamte Management und die Einführung digitalen Lernens in einer Großveranstaltung gelingen.

Den Abschluss des Schwerpunktheftes bildet ein Artikel von Roscher, der zu unserem Erstaunen empirisch erhoben hat, dass sich die Stellenausschreibungen für IT-Manager trotz der in den vorherigen Beiträgen aufgezeigten Veränderungen und großen Herausforderungen kaum verändert haben.

Wie in der HMD üblich, haben wir zwei Spektrumsbeiträge aufgenommen, die das Heft um interessante Erkenntnisse erweitern, die nicht zum Schwerpunkt gehö- 
ren. Hofmeier analysiert auf Basis einer Studie die Resilienz und Digitalisierung in kleinen und mittleren Unternehmen der Lebensmittelproduktion und -logistik, Theobald hinterfragt, ob Künstliche Intelligenz die Produktbeschreibungen für onlineShops effizienter und qualitativ vergleichbar mit menschlich formulierten Texten erstellen kann.

Über ein Jahr Pandemie hat die Welt in vielen Bereichen dramatisch beeinflusst. Die Informationstechnologie hat einen großen Anteil daran, wie wir alle mit den Auswirkungen umgegangen sind. Seit der Einführung des Personal Computers in Wirtschaft, Lehre, Forschung und Administration hat der kleine Virus Covid-19 wohl den größten Impact auf die IT gehabt. Zugegeben können wir in der vorliegenden HMD-Ausgabe nur einen Bruchteil der Herausforderungen und der Lösungen thematisieren, mit der wir konfrontiert wurden. Dennoch zeigen die Erkenntnisse unserer Autoren ganz deutlich auf, dass es deutlich über den Technologie-Einsatz herausgehender Anstrengungen, Kreativität und ja, Menschlichkeit bedarf, um in Krisen $\mathrm{zu}$ bestehen und diese sogar positiv zu nutzen.

Allen Lesern wünschen wir, dass die HMD 340 wertvolle Erfahrungen für ihren beruflichen Alltag vermitteln kann, und Sie Anregungen erhalten, wie Sie trotz, mit und natürlich auch ohne Corona effektiver und effizienter werden. Viel Spaß beim Lesen!

Stefan Reinheimer, Susanne Robra-Bissantz 